\title{
THE CONLEY ATTRACTORS OF AN ITERATED FUNCTION SYSTEM
}

\author{
MICHAEL F. BARNSLEY and ANDREW VINCE ${ }^{\otimes}$ \\ (Received 15 August 2012; accepted 21 February 2013; first published online 11 June 2013)
}

\begin{abstract}
We investigate the topological and metric properties of attractors of an iterated function system (IFS) whose functions may not be contractive. We focus, in particular, on invertible IFSs of finitely many maps on a compact metric space. We rely on ideas of Kieninger [Iterated Function Systems on Compact Hausdorff Spaces (Shaker, Aachen, 2002)] and McGehee and Wiandt ['Conley decomposition for closed relations', Differ. Equ. Appl. 12 (2006), 1-47] restricted to what is, in many ways, a simpler setting, but focused on a special type of attractor, namely point-fibred invariant sets. This allows us to give short proofs of some of the key ideas.
\end{abstract}

2010 Mathematics subject classification: primary 37C70; secondary 28A80.

Keywords and phrases: iterated function system, attractor-repeller.

\section{Introduction}

The subjects of this paper are the attractors of an iterated function system (IFS) on a compact metric space. Iterated function systems are used for the construction of deterministic fractals [2] and have found numerous applications, for example to image compression and image processing [3]. The notion of an attactor of an IFS has historically been linked with the contractive properties of the functions in the IFS, beginning with the work of Hutchinson [10]. If the functions of the IFS are contractions, then the existence of a special type of attractor, a strict attractor, is assured. Moreover, it has recently been shown [1, 17] that, for affine and Möbius IFSs (defined in Section 2), the existence of a strict attractor implies that the functions in the IFS must be contractions. There do exist, however, examples of IFS attractors for which the functions are not contractions, not even contractions with respect to any metric that gives the same topology as the underlying space [6]. In this paper we investigate the topological and metric properties of attractors of a general IFS on a compact metric space for which the functions are not necessarily contractive. We rely on ideas in $[12,14]$, but restricted to what is, in many ways, a simpler setting. The goal is to state and prove basic results about the attractors of a general IFS on a compact metric space, as the groundwork for further research.

(c) 2013 Australian Mathematical Publishing Association Inc. 0004-9727/2013 \$16.00 
There are numerous definitions of an attractor; see [9] and [15, 16], for example. The notion of a strict attractor referred to in the paragraph above is the one that has become standard in the fractal geometry literature. It is natural, however, in the setting of this paper to introduce a more general notion of attractor. For this type of attractor the term Conley attractor is used because it is essentially an extension to a finite set of functions of a notion used so successfully by Conley for a single function [7]. Both the strict attractor and the Conley attractor are defined in Section 2. The attractor block, an essential tool for our investigation of Conley attractors, is the subject of Section 3, the main result being Theorem 3.4 stating that every Conley attractor possesses an attractor block.

Section 4 gives sufficient conditions that guarantee that a Conley attractor is a strict attractor. These sufficient conditions involve both contractive properties of the functions in the IFS and the existence of a natural addressing function for the points of the attractor. The notion of fibring plays a role; the thesis of Kieninger [12] has an extensive discussion of the subject of fibring.

In the case that the functions in the IFS $\mathcal{F}$ are invertible, there is a duality between the action of the IFSs $\mathcal{F}$ and $\mathcal{F}^{*}$, where $\mathcal{F}^{*}$ consists of the inverses of the maps in $\mathcal{F}$. This leads to the notion of an attractor-repeller pair $\left(A, A^{*}\right)$, where $A$ is a Conley attractor of $\mathcal{F}$ and $A^{*}$ is the 'dual' disjoint Conley attractor of $\mathcal{F}^{*}$. The main theorem is as follows, where the notion of basin is given in Definition 2.2.

THEOREM 1.1. Let $\mathcal{F}$ be an invertible IFS on a compact metric space $\mathbb{X}$. If A is a Conley attractor of $\mathcal{F}$ with basin $B$, then $A^{*}:=\mathbb{X} \backslash B$ is a repeller of $\mathcal{F}$ with basin $\mathbb{X} \backslash A$.

For the dynamics of a single function, this plays a significant role in Conley's index theory [7] and has been extended to the context of 'closed relations' by McGehee and Wiandt $[13,14]$. In general, an invertible IFS can have many Conley attractor-repeller pairs. The second main result in Section 5 is Theorem 5.5, which relates the structure of these Conley attractor-repeller pairs to the dynamics of the IFS $\mathcal{F}$, more specifically to the set of chain-recurrent points of $\mathcal{F}$.

The last section provides some examples of the properties described in the paper.

\section{Attractors}

Unless otherwise stated, throughout this section $(\mathbb{X}, d)$ is a complete metric space. The closure of a set $B$ is denoted $\bar{B}$ and the interior by $B^{o}$.

Definition 2.1. If $f_{n}: \mathbb{X} \rightarrow \mathbb{X}, n=1,2, \ldots, N$, are continuous functions, then $\mathcal{F}=$ $\left(\mathbb{X} ; f_{1}, f_{2}, \ldots, f_{N}\right)$ is called an iterated function system (IFS). If each of the maps $f \in \mathcal{F}$ is a homeomorphism then $\mathcal{F}$ is said to be invertible, and the notation $\mathcal{F}^{*}:=$ $\left(\mathbb{X} ; f_{1}^{-1}, f_{2}^{-1}, \ldots, f_{N}^{-1}\right)$ is used.

Subsequently in this paper we refer to some special cases of an IFS. For an affine IFS we have $\mathbb{X}=\mathbb{R}^{n}$ and the functions in the IFS are affine functions of the form $f(x)=A x+a$, where $A$ is an $n \times n$ matrix and $a \in \mathbb{R}^{n}$. For a projective IFS 
we have $\mathbb{X}=\mathbb{R} \mathbb{P}^{n}$, real projective space, and the functions in the IFS are projective functions of the form $f(x)=A x$, where $A$ is an $(n+1) \times(n+1)$ matrix and $x$ is given by homogeneous coordinates. For a Möbius IFS we have $\mathbb{X}=\widehat{\mathbb{C}}=\mathbb{C} \cup\{\infty\}$, the extended complex plane, and the functions are Möbius functions of the form $f(z)=$ $(a z+b) /(c z+d)$, where $a d-b c=1$ and $a, b, c, d$ are complex numbers. Möbius functions may equivalently be considered to act on the Riemann sphere or the complex projective line.

By a slight abuse of terminology we use the same symbol $\mathcal{F}$ for the IFS, the set of functions in the IFS, and for the following mapping. Letting $2^{\mathbb{X}}$ denote the collection of subsets of $\mathbb{X}$, define $\mathcal{F}: 2^{\mathbb{X}} \rightarrow 2^{\mathbb{X}}$ by

$$
\mathcal{F}(B)=\bigcup_{f \in \mathcal{F}} f(B)
$$

for all $B \in 2^{\mathbb{X}}$. Let $\mathbb{H}=\mathbb{H}(\mathbb{X})$ be the set of nonempty compact subsets of $\mathbb{X}$. Since $\mathcal{F}(\mathbb{H}) \subseteq \mathbb{H}$ we can also treat $\mathcal{F}$ as a mapping $\mathcal{F}: \mathbb{H} \rightarrow \mathbb{H}$. Let $d_{\mathbb{H}}$ denote the Hausdorff metric on $\mathbb{H}$, which can be defined as follows. Using the notation

$$
S_{r}=\left\{y \in \mathbb{X}: d_{\mathbb{X}}(x, y)<r \text { for some } x \in S\right\}
$$

with $S \subset \mathbb{X}$ and $r>0$, the Hausdorff metric $d_{\mathbb{H}}$ (see for example [8, page 66]) is

$$
d_{\mathbb{H}}(B, C)=\inf \left\{r>0: B \subset C_{r} \text { and } C \subset B_{r}\right\}
$$

for all $B, C \in \mathbb{H}$. Under various conditions the map $\mathcal{F}: \mathbb{H} \rightarrow \mathbb{H}$ is continuous with respect to $d_{\mathbb{H}}$. This occurs, for example, when the metric space $\mathbb{X}$ is compact or when each $f \in \mathcal{F}$ is Lipschitz, see [5]. It was also proved to be true when $\mathbb{X}$ is a complete metric space; see [4].

For $B \subset \mathbb{X}$ and $k \in \mathbb{N}:=\{1,2, \ldots\}$, let $\mathcal{F}^{k}(B)$ denote the $k$-fold composition of $\mathcal{F}$, the union of $f_{i_{1}} \circ f_{i_{2}} \circ \cdots \circ f_{i_{k}}(B)$ over all finite words $i_{1} i_{2} \ldots i_{k}$ of length $k$. Define $\mathcal{F}^{0}(B)=B$. A strict attractor is defined as follows. This concept coincides with the standard notion of an IFS attractor in much of the literature on IFS theory.

Definition 2.2. A nonempty set $A \in \mathbb{H}(\mathbb{X})$ is said to be a strict attractor of the IFS $\mathcal{F}$ if:

(i) $\mathcal{F}(A)=A$; and

(ii) there is an open set $U \subset \mathbb{X}$ such that $A \subset U$ and $\lim _{k \rightarrow \infty} \mathcal{F}^{k}(S)=A$ for all $S \in \mathbb{H}(U)$, where the limit is with respect to the Hausdorff metric.

The largest open set $U$ such that (ii) is true is called the basin of the strict attractor $A$ of the IFS $\mathcal{F}$.

The following less restrictive (see statement (4) of Proposition 2.4) definition of an attractor $A$ is also used in this paper. Called a Conley attractor, it generalises a notion of attractor due to Conley [7] that has proved useful in the study of the dynamics of a single function. The definition states, in the case of nonempty attractors, that there is an open set $U$ containing $A$ whose closure converges, in the Hausdorff sense, to $A$. 
Definition 2.3. A compact set $A$ is said to be a Conley attractor of the IFS $\mathcal{F}$ if there is an open set $U$ such that $A \subset U$ and

$$
A=\lim _{k \rightarrow \infty} \mathcal{F}^{k}(\bar{U})
$$

The basin of $A$ is the union of all open sets $U$ that satisfy the above definition.

The empty set is always a Conley attractor of an IFS and $\mathbb{X}$ is a Conley attractor if $\mathcal{F}$ contains at least one surjective function. Example 6.1 provides an IFS with infinitely many Conley attractors.

Proposition 2.4. Let $\mathcal{F}$ be an IFS on a compact metric space.

(1) If $A$ is a Conley attractor of $\mathcal{F}$, then $\mathcal{F}(A)=A$.

(2) If $A$ is a Conley attractor of $\mathcal{F}$ with basin $B$ and $S$ is any compact set such that $A \subseteq S \subset B$, then $\lim _{k \rightarrow \infty} \mathcal{F}^{k}(S)=A$.

(3) If $A$ and $A^{\prime}$ are Conley attractors of an IFS $\mathcal{F}$, then $A \cup A^{\prime}$ and $A \cap A^{\prime}$ are also Conley attractors.

(4) If $A$ is a strict attractor with basin B, then $A$ is a Conley attractor with basin B.

Proof. Concerning statement (1),

$$
\mathcal{F}(A)=\mathcal{F}\left(\lim _{k \rightarrow \infty} \mathcal{F}^{k}(\bar{U})\right)=\lim _{k \rightarrow \infty} \mathcal{F}^{k+1}(\bar{U})=A .
$$

Statement (2) follows from statement (1) and the fact that $S$, being compact, is contained in the union of finitely many open sets $U$ such that $A=\lim _{k \rightarrow \infty} \mathcal{F}^{k}(\bar{U})$.

Concerning statement (3), in Definition 2.3, let $U$ be the open set for $A$ and $U^{\prime}$ the open set for $A^{\prime}$. Then $U \cup U^{\prime}$ and $U \cap U^{\prime}$ are the required open sets for $A \cup A^{\prime}$ and $A \cap A^{\prime}$.

Concerning statement (4), let $U$ be an open set containing $A$ such that $\bar{U} \subset B$. Then $U$ satisfies the conditions in the definition of a Conley attractor. Let $B^{\prime}$ denote the basin of $A$ as given in Definition 2.3. To show that $B^{\prime}=B$, first note that $\bigcup\{U: A \subset$ $U \subset \bar{U} \subset B\}=B$. Therefore, $B \subseteq B^{\prime}$. Moreover, if $S$ is any compact subset of $B^{\prime}$, then there is an open set $U$ such that $A \cup S \subset U \subset \bar{U} \subset B^{\prime}$. Since $\lim _{k \rightarrow \infty} \mathcal{F}^{k}(\bar{U})=A$, there is a $k$ such that $\mathcal{F}^{k}(S) \subset B$. It follows that $\lim _{k \rightarrow \infty} \mathcal{F}^{k}(S)=A$.

REMARK 2.5. Conley's concept of an attractor for one function is usually expressed as an $\omega$-limit. Although it is slightly more complicated to do so, our definition of Conley attractor of an IFS could be defined in a similar manner. Let

$$
\mathcal{K}(S):=\left\{Y: Y \text { closed, } \mathcal{F}(Y) \subset Y \text {, and } \mathcal{F}^{n}(S) \subset Y \text { for some } n \geq 0\right\}
$$

The $\omega$-limit set of a set $S$ under $\mathcal{F}$ is the set

$$
\omega(S)=\bigcap \mathcal{K}(S)
$$


In our context, it can be proved that this is equivalent to

$$
\omega(S):=\bigcap_{K \geq 1} \overline{\bigcup_{k \geq K} \mathcal{F}^{k}(S),}
$$

which is referred to as the strict $\omega$-limit. Omitting the proof, we state that a set $A \subset \mathbb{X}$ is a Conley attractor of the IFS $\mathcal{F}$ in the sense of Definition 2.3 if and only if:

(i) $A=\omega(U)$ for some open subset $U$ of $\mathbb{X}$; and

(ii) $A \subset U$.

Furthermore, the largest open set $B \subset \mathbb{X}$ such that $\omega(\{x\}) \subset A$ for all $x \in B$ is the basin of $A$. It follows from the equivalence of the two definitions that the Hausdorff limit in Definition 2.3 exists if and only if $\overline{\bigcup_{k \geq K} \mathcal{F}^{k}(U)} \subset U$ for some $K$.

In the following lemma we use the notation $\vec{d}(X, Y)=\max _{x \in X} \min _{y \in Y} d(x, y)$ for compact sets $X$ and $Y$. The lemma states that the basin of a Conley attractor $A$ consists of those points whose images under iterates of $\mathcal{F}$ get arbitrarily close to $A$.

Lemma 2.6. If $A$ is a Conley attractor of an IFS $\mathcal{F}$, then the basin of $A$ is

$$
B=\left\{x: \lim _{k \rightarrow \infty} \vec{d}\left(\mathcal{F}^{k}(x), A\right)=0\right\}=\{x: \omega(\{x\}) \subset A\} .
$$

Proof. Let $B$ denote the basin for $A$ and $B^{\prime}$ the set in (2.1). It follows from the definitions that $B \subseteq B^{\prime}$.

Let $U$ be an open set containing $A$ such that $\lim _{k \rightarrow \infty} \mathcal{F}^{k}(\bar{U})=A$. To prove that $B^{\prime} \subseteq B$, it suffices to show that, for any $x \in B^{\prime}$, there is an open neighbourhood $N$ of $x$ such that, if $U^{\prime}=U \cup N$, then $\lim _{k \rightarrow \infty} \mathcal{F}^{k}\left(\bar{U}^{\prime}\right)=A$, and hence $x \in B$. To show that such a neighbourhood $N$ exists, let $\epsilon>0$ be such that $\left\{x: \min _{a \in A} d(x, a)<\epsilon\right\} \subset U$. Then there is a $K$ such that if $k \geq K$, then $\lim _{k \rightarrow \infty} \vec{d}\left(\mathcal{F}^{k}(x), A\right)<\epsilon / 2$. By the continuity of the functions in $\mathcal{F}$, there is a $\delta>0$ such that, if $d(x, y)<\delta$, then $d(g(x), g(y))<\epsilon / 2$ for all $g \in \mathcal{F}^{K}$. Therefore, $\mathcal{F}^{K}(N) \subset U$ and $\lim _{k \rightarrow \infty} \mathcal{F}^{k}\left(\bar{U}^{\prime}\right)=\lim _{k \rightarrow \infty} \mathcal{F}^{k}(\bar{U})=A$.

\section{Attractor blocks}

Definition 3.1. If $\mathcal{F}$ is an IFS on a compact metric space $\mathbb{X}$, then $Q \subset \mathbb{X}$ is called an attractor block with respect to $\mathcal{F}$ if $\mathcal{F}(\bar{Q}) \subset Q^{\circ}$.

The following proposition is easy to verify.

Proposition 3.2. If $Q$ is an attractor block with respect to the IFS $\mathcal{F}$ on a compact metric space $\mathbb{X}$, then $\lim _{k \rightarrow \infty} \mathcal{F}^{k}(\bar{Q})=\bigcap_{k \rightarrow \infty} \mathcal{F}^{k}(\bar{Q})$ exists and is a Conley attractor of $\mathcal{F}$.

In light of Proposition 3.2 we formulate the following definition.

Definition 3.3. If $Q$ is an attractor block and

$$
A=\bigcap_{k \rightarrow \infty} \mathcal{F}^{k}(\bar{Q})
$$


is the corresponding Conley attractor, then $Q$ is called an attractor block for $A$ with respect to $\mathcal{F}$.

The basin of a Conley attractor is not, in general, an attractor block. For, if $B$ is the basin for a Conley attractor then, using the continuity of $\mathcal{F}$, we have $\mathcal{F}(\bar{B})=\bar{B}$. Therefore, unless $\bar{B}$ is open, $\mathcal{F}(\bar{B})$ is not contained in the interior of $B$, and so it cannot be an attractor block. Nevertheless, the following theorem tells us that every Conley attractor has a corresponding attractor block for it.

THeOREM 3.4. If $\mathcal{F}$ is an IFS on a compact metric space, $A$ is a Conley attractor of $\mathcal{F}$, and $\mathcal{N}$ is a neighbourhood of $A$, then there is an attractor block for A contained in $\mathcal{N}$.

Proof. The proof makes use of the function $\mathcal{F}^{-1}(X)=\{x \in \mathbb{X}: f(x) \in X$ for all $f \in \mathcal{F}\}$. Note that $\mathcal{F}^{-1}$ takes open sets to open sets, $X \subset\left(\mathcal{F}^{-1} \circ \mathcal{F}\right)(X)$ and $\left(\mathcal{F} \circ \mathcal{F}^{-1}\right)(X) \subset X$ for all $X$.

Let $U^{\prime}$ denote an open set containing $A$ such that $A=\lim _{k \rightarrow \infty} \mathcal{F}^{k}\left(\overline{U^{\prime}}\right)$, and let $U=$ $U^{\prime} \cap \mathcal{N}$. Let $V$ be an open set such that $A \subset V$ and $\bar{V} \subset U$. Since $A=\lim _{k \rightarrow \infty} \mathcal{F}^{k}(\bar{V})$ by statement (2) of Proposition 2.4, there is an integer $m$ such that $\overline{\mathcal{F}^{k}(V)} \subset V$ for all $k>m$. Define $V_{k}, k=0,1, \ldots, m$, recursively, going backwards from $V_{m}$ to $V_{0}$, as follows. Let $V_{m}=V$ and for $k=m-1, \ldots, 2,1,0$, let $V_{k}=V \cap \mathcal{F}^{-1}\left(V_{k+1}\right)$. If $O=V_{0}$, then $O$ has the following properties:

(1) $O$ is open;

(2) $A \subset O$;

(3) $\mathcal{F}^{k}(O) \subset V$ for all $k \geq 0$.

Property (2) follows from the fact that $A \subset \mathcal{F}^{-1}(A)$. Property (3) follows from the facts that $\mathcal{F}^{k}(O) \subset V_{k} \subset V$ for $0 \leq k \leq m$, and $\mathcal{F}^{k}(O) \subset \mathcal{F}^{k}(V) \subset V$ for all $k>m$.

Since, by statement (2) of Proposition 2.4,A $=\lim _{k \rightarrow \infty} \mathcal{F}^{k}(\bar{O})$, there is an integer $K$ such that $\mathcal{F}^{K}(\bar{O})=\overline{\mathcal{F}^{K}(O)} \subset O$. Let $O_{k}, k=0,1, \ldots, K$, be defined recursively, going backwards from $O_{K}$ to $O_{0}$, as follows. Let $O_{K}$ be an open set such that $\mathcal{F}^{K}(\bar{O}) \subset O_{K} \subset O$, and for $k=K-1, \ldots, 2,1,0$, let $O_{k}$ be an open set such that:

(1) $\mathcal{F}^{k}(\bar{O}) \subset O_{k} \subset U$; and

(2) $\mathcal{F}\left(\bar{O}_{k}\right) \subset O_{k+1}$.

To verify that a set $O_{k}$ with these properties exists, assume that $O_{k}, k \geq 1$, has been chosen with properties (1) and (2) and note that $\mathcal{F}^{k-1}(\bar{O}) \subset \mathcal{F}^{-1}\left(\mathcal{F}^{k}(\bar{O})\right) \subset \mathcal{F}^{-1}\left(O_{k}\right)$ and $\mathcal{F}^{k-1}(\bar{O}) \subset \bar{V} \subset U$. Now choose $O_{k-1}$ to be an open set such that $\mathcal{F}^{k-1}(\bar{O}) \subset O_{k-1}$ and $\overline{O_{k-1}} \subset U \cap \mathcal{F}^{-1}\left(O_{k}\right) \subset U$. The last inclusion implies $\mathcal{F}\left(\bar{O}_{k-1}\right) \subset O_{k}$.

We claim that

$$
Q=\bigcup_{k=0}^{K-1} O_{k}
$$


is an attractor block for $A$. Since $A=\mathcal{F}^{k}(A) \subset \mathcal{F}^{k}(O) \subset O_{k}$ for each $k$, we have $A \subset Q$. Clearly, $Q$ is an open set such that $Q \subset U \subset \mathcal{N}$. Hence $A=\lim _{k \rightarrow \infty} \mathcal{F}^{k}(\bar{Q})$, and lastly,

$$
\mathcal{F}(\bar{Q})=\bigcup_{k=0}^{K-1} \mathcal{F}\left(\overline{O_{k}}\right) \subset \bigcup_{k=1}^{K} O_{k}=\bigcup_{k=1}^{K-1} O_{k} \cup O_{K} \subset Q \cup O \subset Q \cup O_{0} \subset Q .
$$

This concludes the proof.

\section{Sufficient conditions for a Conley attractor to be a strict attractor}

In this section, $A$ is a Conley attractor of an IFS $\mathcal{F}$ on a compact metric space and $B$ is the basin of $A$. Under certain conditions $A$ is guaranteed to be a strict attractor. In particular, contractive properties of the functions in $\mathcal{F}$ or of the fibres of $\mathcal{F}$ may force this.

Definition 4.1. An IFS $\mathcal{F}$ on a metric space $(\mathbb{X}, d)$ is said to be contractive if there is a metric $\hat{d}$ inducing the same topology on $\mathbb{X}$ as the metric $d$ with respect to which the functions in $\mathcal{F}$ are strict contractions, that is, there exists $\lambda \in[0,1)$ such that $\hat{d}_{\mathbb{X}}(f(x), f(y)) \leq \lambda \hat{d}_{\mathbb{X}}(x, y)$ fo all $x, y \in \mathbb{X}$ and for all $f \in \mathcal{F}$.

A classical result of Hutchinson [10], a result marking the origin of the concept of an iterated function system, states that if $\mathcal{F}$ is contractive on a complete metric space $\mathbb{X}$, then $\mathcal{F}$ has a unique strict attractor with basin $\mathbb{X}$. The corollary below follows from Hutchinson's result.

Corollary 4.2. Let A be a Conley attractor of an IFS $\mathcal{F}$ on a metric space and let $B$ be the basin of $A$. If $\mathcal{F}$ is contractive on $B$, then $A$ is a strict attractor of $\mathcal{F}$ with basin $B$.

Proof. If $S$ is any compact subset of $B$ containing $A$, then $S$ is a complete metric space. Hutchinson's result implies that there is a unique strict attractor $A^{\prime}$ in $B$ and that $B \subseteq B^{\prime}$, where $B^{\prime}$ is the attractor of $A^{\prime}$. It only remains to show that $A^{\prime}=A$ and $B^{\prime}=B$. Let $U$ be an open set containing $A$ and $A^{\prime}$ and such that $\bar{U} \subset B$. Then by the definitions of the Conley and strict attractor $A^{\prime}=\lim _{k \rightarrow \infty} \mathcal{F}^{k}(\bar{U})=A$. Moreover, if $A \subset U \subset \bar{U} \subset B^{\prime}$, then by the definition of strict attractor we have $\lim _{k \rightarrow \infty} \mathcal{F}^{k}(\bar{U})=A^{\prime}=A$. Therefore, $B^{\prime} \subseteq B$.

THeOREM 4.3. If F is an affine IFS or a Möbius IFS with a nontrivial Conley attractor $A$, then $A$ is a strict attractor, and it is unique.

Proof. The theorem for the affine case follows from Theorem 3.4, [1, Theorem 1.1], and Corollary 4.2. According to Theorem 3.4, the Conley attractor $A$ has an attractor block $Q$. According to [1, Theorem 1.1], if there is a compact set $Q$ such that $\mathcal{F}(Q) \subset Q^{o}$, then $\mathcal{F}$ is contractive on $\mathbb{R}^{n}$. By Corollary 4.2, the Conley attractor $A$ is a strict attractor. By Hutchinson's theorem, there is a unique strict attractor in $\mathbb{R}^{n}$.

The proof in the Möbius case is the same except that [17, Theorem 1.1] is used in place of [1, Theorem 1.1]. 
The analogous result to Theorem 4.3 fails for a projective IFS on the projective plane $\mathbb{R} \mathbb{P}^{2}$. See Example 6.2 in Section 6.

The next theorem generalises Corollary 4.2 by replacing the contractivity condition by a weaker condition called the point-fibred condition. Let $\Omega$ denote the set of all infinite sequences $\left\{\sigma_{k}\right\}_{k=1}^{\infty}$ of symbols belonging to the alphabet $\{1, \ldots, N\}$. A typical element of $\Omega$ can be denoted as $\sigma=\sigma_{1} \sigma_{2} \sigma_{3} \ldots$ With

$$
d_{\Omega}(\sigma, \omega)= \begin{cases}0 & \text { when } \sigma=\omega, \\ 2^{-k} & \text { when } k \text { is the least index for which } \sigma_{k} \neq \omega_{k},\end{cases}
$$

$\left(\Omega, d_{\Omega}\right)$ is a compact metric space called a code space. The topology on $\Omega$ induced by the metric $d_{\Omega}$ is the same as the product topology that is obtained by treating $\Omega$ as the infinite product space $\{1, \ldots, N\}^{\infty}$. For an IFS $\mathcal{F}$ and $\sigma \in \Omega$, we use the shorthand notation

$$
f_{\sigma \mid k}=f_{\sigma_{1}} \circ f_{\sigma_{2}} \circ \cdots \circ f_{\sigma_{k}} .
$$

The limit, where $K$ is a compact set,

$$
\pi_{\mathcal{F}}(\sigma, K):=\lim _{k \rightarrow \infty} f_{\sigma \mid k}(K),
$$

if it exists, is referred to as a fibre of the IFS $\mathcal{F}$.

Definition 4.4. An IFS $\mathcal{F}$ is point-fibred on a set $B$ if

$$
\pi_{\mathcal{F}}(\sigma):=\lim _{k \rightarrow \infty} f_{\sigma \mid k}(K)
$$

exists for all $\sigma \in \Omega$ and, for each $\sigma$, is independent of $K \subset B$.

Point-fibred IFSs are important because they possess natural addressing maps, or 'coordinates', related to the functions of the IFS. The addresses are infinite strings in the alphabet $\{1,2, \ldots, N\}$, where $N$ is the number of functions in the IFS. The precise definition is as follows.

Definition 4.5. Let $\mathcal{F}$ be an IFS on a metric space $\mathbb{X}$ consisting of $N$ continuous functions. If $\pi: \Omega \rightarrow \mathbb{X}$ is a continuous mapping such that the following diagram commutes for all $n \in\{1,2, \ldots, N\}$

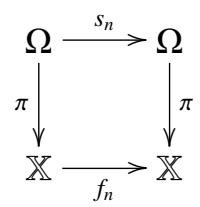

where $s_{n}$ is the inverse shift defined by $s_{n}(\sigma)=n \sigma$, then $\pi$ is called a coding map for $\mathcal{F}$. The map $\pi$ is also referred to as an addressing function.

THEOREM 4.6. If $F$ is a point-fibred iterated function system on a compact metric space $\mathbb{X}$, then:

(1) $\mathcal{F}: \mathbb{H}(\mathbb{X}) \rightarrow \mathbb{H}(\mathbb{X})$ has a unique fixed point $A \in \mathbb{H}(\mathbb{X})$, that is $\mathcal{F}(A)=A$;

(2) $A$ is the unique strict attractor of $\mathcal{F}$ in $\mathbb{X}$; 
(3) the basin of $A$ is $\mathbb{X}$;

(4) the map $\pi_{\mathcal{F}}: \Omega \rightarrow \mathbb{X}$ given by $\pi_{\mathcal{F}}(\sigma):=\lim _{k \rightarrow \infty} f_{\sigma \mid k}(K)$ is a coding map;

(5) the range of the coding map $\pi_{\mathcal{F}}$ is $A$, that is $\pi(\Omega)=A$.

Proof. The theorem follows from [12, Proposition 4.4.2, page 107, Proposition 3.4.4, page 77].

If $\mathcal{F}$ is an affine IFS, then the converse of statement (4) in Theorem 4.6 is true. A coding map of an affine IFS must be of the form (4.1). The following result appears in [1, Theorem 7.2], where the affine hull of a set is the smallest affine subspace containing the set.

THEOREM 4.7. If an affine IFS $\mathcal{F}$ on $\mathbb{R}^{n}$ has a coding map $\pi$ and the affine hull of $A:=\pi(\Omega)$ equals $\mathbb{R}^{n}$, then $\mathcal{F}$ is point-fibred on $\mathbb{R}^{n}$. Moreover $A$ is the strict attractor of $\mathcal{F}$.

The following generalisation of Corollary 4.2 follows from Theorem 4.6 in exactly the same way as Corollary 4.2 followed from Hutchinson's theorem. It is a generalisation because it easy to show that if $\mathcal{F}$ is contractive on a complete metric space $\mathbb{X}$, then $\mathcal{F}$ is point-fibred on $\mathbb{X}$. Research on when the property of being pointfibred implies that the IFS is contractive is ongoing; see [11].

Corollary 4.8. Let $\mathcal{F}$ be an IFS on a compact metric space with a Conley attractor $A$ and basin $B$. If $\mathcal{F}$ is point-fibred on $B$, then $A$ is a strict attractor of $\mathcal{F}$ with basin $B$.

\section{Attractor-repeller pairs}

In this section it is assumed that the iterated function system is invertible.

Definition 5.1. A set $R \subset \mathbb{X}$ is said to be a repeller of the invertible IFS $\mathcal{F}$ if $R$ is a Conley attractor of $\mathcal{F}^{*}=\left\{f^{-1}: f \in \mathcal{F}\right\}$. The basin of a repeller of $\mathcal{F}$ is the basin for the corresponding Conley attractor of $\mathcal{F}^{*}$.

THeOREM 5.2. Let $\mathcal{F}$ be an invertible IFS on a compact metric space $\mathbb{X}$. If A is a Conley attractor of $\mathcal{F}$ with basin $B$, then $A^{*}:=\mathbb{X} \backslash B$ is a repeller of $\mathcal{F}$ with basin $\mathbb{X} \backslash A$.

Proof. By Theorem 3.4, there is an attractor block $Q$ for $A$ with respect to $\mathcal{F}$. It is easy to verify that the complement $Q^{*}:=\mathbb{X} \backslash Q$ is an attractor block with respect to $\mathcal{F}^{*}$. Let $A^{*}=\lim _{k \rightarrow \infty} \mathcal{F}^{* k}\left(\overline{Q^{*}}\right)$ be the corresponding topological Conley attractor as guaranteed by Proposition 3.2.

It is now sufficient to show that the basin $B$ of $A$ is $\mathbb{X} \backslash A^{*}$, and to do this Lemma 2.6 is used. If $x \in Q$, then $\lim _{k \rightarrow \infty} \vec{d}\left(\mathcal{F}^{k}(x), A\right)=0$ because $\lim _{k \rightarrow \infty} f^{k}(\bar{Q})=A$. Therefore, $x \in B$. Now let $x \in Q^{*} \backslash A^{*}$. If $\mathcal{F}^{k}(x) \subset Q$ for some $k$, then again

$$
\lim _{k \rightarrow \infty} \vec{d}\left(\mathcal{F}^{k}(x), A\right)=0
$$

and the proof is complete. So, by way of contradiction, assume that $\mathcal{F}^{k}(x)$ is not a subset of $Q$ for any $k$. Then there is a set $X=\left\{x_{k}\right\}$ such that $x_{k} \in \mathcal{F}^{k}(x)$ and $x_{k} \in Q^{*}$. 
In this case $x \in \mathcal{F}^{* k}(X) \subset \mathcal{F}^{* k}\left(Q^{*}\right)$ for all $k$. Since $\lim _{k \rightarrow \infty} \mathcal{F}^{* k}\left(\overline{Q^{*}}\right)=A^{*}$, this implies that $x \in A^{*}$, a contradiction.

It only remains to show that $B \cap A^{*}=\emptyset$. Let $x=x_{0} \in A^{*}$. Because $\mathcal{F}^{*}\left(A^{*}\right)=A^{*}$, by statement (1) of Proposition 2.4, there is an $x_{1} \in A^{*}$ and an $f_{1} \in \mathcal{F}$ such that $f_{1}^{-1}\left(x_{1}\right)=x_{0}$, that is, $x_{1}=f_{1}(x)$. For the same reason there is an $x_{2} \in A^{*}$ and an $f_{2} \in \mathcal{F}$ such that $f_{2}^{-1}\left(x_{2}\right)=x_{1}$, that is, $x_{2}=\left(f_{2} \circ f_{1}\right)(x)$. Continuing in this way, it is clear that $\mathcal{F}^{k}(x) \cap A^{*} \neq \emptyset$ for all $k>0$, which implies by Lemma 2.6 that $x$ does not lie in $B$.

Definition 5.3. If $\mathcal{F}$ is an invertible IFS on a compact metric space $\mathbb{X}$ and $A$ is a Conley attractor of $\mathcal{F}$ with basin $B$, then the set

$$
A^{*}:=\mathbb{X} \backslash B
$$

is called the dual repeller of $A$.

Examples of attractor-repeller pairs are shown in Section 6. The notion of a chain for an IFS is based on the notion of a chain for a single function [7].

Definition 5.4. Let $\varepsilon>0$ and let $\mathcal{F}$ be an IFS on $\mathbb{X}$. An $\varepsilon$-chain for $\mathcal{F}$ is a sequence of points $\left\{x_{i}\right\}_{i=0}^{n}, n>0$, in $\mathbb{X}$ such that for each $i \in\{0,1,2, \ldots, n-1\}$ there is an $f \in \mathcal{F}$ such that $d\left(x_{i+1}, f\left(x_{i}\right)\right)<\varepsilon$. A point $x \in \mathbb{X}$ is chain-recurrent for $\mathcal{F}$ if, for every $\varepsilon>0$, there is an $\varepsilon$-chain $\left\{x_{i}\right\}_{i=0}^{n}$ for $\mathcal{F}$ such that $x_{0}=x_{n}=x$. The set of all chain-recurrent points for $\mathcal{F}$ is denoted by $\mathcal{R}:=\mathcal{R}(\mathcal{F})$.

We refer to the following as the Conley-McGehee-Wiandt (CMW) theorem due to previous versions in a non IFS context.

Theorem 5.5 (CMW). Let $\mathcal{F}$ be an invertible IFS on a compact metric space $\mathbb{X}$. If $\mathcal{U}$ denotes the set of Conley attractors of $\mathcal{F}$ and $\mathcal{R}$ denotes the set of chain-recurrent points of $\mathcal{F}$, then

$$
\mathcal{R}=\bigcap_{A \in \mathcal{U}}\left(A \cup A^{*}\right) .
$$

Proof. First assume that $x \notin \bigcap_{A \in \mathcal{U}}\left(A \cup A^{*}\right)$. Then there is a Conley attractor $A$ such that $x \notin A \cup A^{*}$. By Theorem 5.2, the point $x$ lies in the basin of $A$. According to Theorem 3.4, there is a closed attractor block $Q$ for $A$ with respect to $\mathcal{F}$ such that $x \notin Q$. Since $x$ lies in the basin of $A$, there is an integer $K$ such that $\mathcal{F}^{k}(x) \subset Q^{o}$ for all $k \geq K$. Since $\mathcal{F}(Q) \subset Q^{o}$,

$$
d:=\min \left\{d\left(y, y^{\prime}\right): y \in \mathcal{F}(Q), y^{\prime} \in \partial Q\right\}>0 .
$$

We must show that $x \notin R$. By way of contradiction, assume that $x$ is chainrecurrent and that $\left\{x_{i}\right\}_{i=0}^{n}$ is an $\epsilon$-chain with $x_{0}=x_{n}=x$ and $\epsilon<d$. We may assume, by repeating the chain if necessary, that $n \geq K$. Since $\mathcal{F}^{k}(x) \subset Q^{o}$ for all $k \geq K$ and by the continuity of the functions in $\mathcal{F}$, if $\epsilon$ is sufficiently small, say $\epsilon=\epsilon_{0}$, then $x_{K} \in Q$. Now $d\left(x_{K+1}, f\left(x_{K}\right)\right)<\epsilon_{0}<d$ for some $f \in \mathcal{F}$. Therefore, $f\left(x_{K}\right) \in Q^{o}$ implies that $x_{K+1} \in Q$. Repeating this argument shows that $x_{i} \in Q$ for all $i \geq K$. From the first paragraph in the proof $x \notin Q$ and by the above $x=x_{n} \in Q$, a contradiction. 
Conversely, assume that $x \notin \mathcal{R}$. Then there is an $\epsilon>0$ such that no $\epsilon$-chain starts and ends at $x$. Let $U$ denote the set of all points $y$ such that there is an $\epsilon$-chain from $x$ to $y$. Notice that: (1) $x \notin U$; (2) $U$ is an open set; and (3) $\mathcal{F}(\bar{U}) \subset U$. Therefore, $A:=\lim _{k \rightarrow \infty} \mathcal{F}^{k}(\bar{U})$ is a Conley attractor with $x \notin A$. Since $\mathcal{F}(x) \subset U$ and $A:=\lim _{k \rightarrow \infty} \mathcal{F}^{k}(\bar{U})$, the point $x$ lies in the basin of $\mathcal{F}$, and therefore $x \notin A^{*}$ by Theorem 5.2. So $x \notin A \cup A^{*}$.

\section{Examples}

ExAmple 6.1. This is an example of an IFS with infinitely many Conley attractors. Let $n$ be an integer and consider the IFS on $\mathbb{R}$ consisting of the single function

$$
f(x)= \begin{cases}x^{2}-2 n x+\left(n^{2}+n\right) & \text { if } n \leq x<n+1, n \geq 0, \\ -x^{2}+2(n+1) x-\left(n^{2}+n\right) & \text { if } n \leq x<n+1, n<0 .\end{cases}
$$

For all integers $m, n \geq 0$, the interval $[-m, n]$ is a Conley attractor with basin $(-m-1$, $n+1)$.

ExAmple 6.2. This example shows that the analogous result to Theorem 4.3 fails for a projective IFS on the projective plane $\mathbb{R} \mathbb{P}^{2}$. Consider the IFS consisting of a single projective function $f$ represented by the matrix

$$
\left(\begin{array}{lll}
2 & 0 & 0 \\
0 & 1 & 0 \\
0 & 0 & 1
\end{array}\right)
$$

The line in $\mathbb{R} \mathbb{P}^{2}$ corresponding to the $y, z$-plane in $\mathbb{R}^{3}$ is a Conley attractor, but it is not a strict attractor.

Example 6.3 (Contractive IFS). Figure 1 shows the attractor-repeller pair of a Möbius IFS whose space is the Riemann sphere. Since this IFS is contractive it has a unique nontrivial strict attractor-repeller pair. The basin of the attractor is the complement of the repeller, and the basin of the repeller is the complement of the attractor. For further details on such Möbius examples, see [17].

EXAMPLE 6.4 (Noncontractive IFS). A noncontractive IFS may have no nontrivial Conley attractor. For example, the IFS on the unit circle centred at the origin of the complex plane consisting of the single function $f(z)=i z$ clearly has no Conley attractor.

Example 6.1 is a noncontractive IFS with infinitely many Conley attractors.

The following projective IFS, whose space is the projective plane, is noncontractive but has a unique nontrivial strict attractor $A$ shown in Figure 2. The projective plane is depicted as a disc with antipodal points identified. This IFS consists of two functions given in matrix form by

$$
f_{1}=\left(\begin{array}{ccc}
41 & -19 & 19 \\
-19 & 41 & 19 \\
19 & 19 & 41
\end{array}\right) \quad \text { and } \quad f_{2}=\left(\begin{array}{ccc}
-10 & -1 & 19 \\
-10 & 21 & 1 \\
10 & 10 & 10
\end{array}\right)
$$




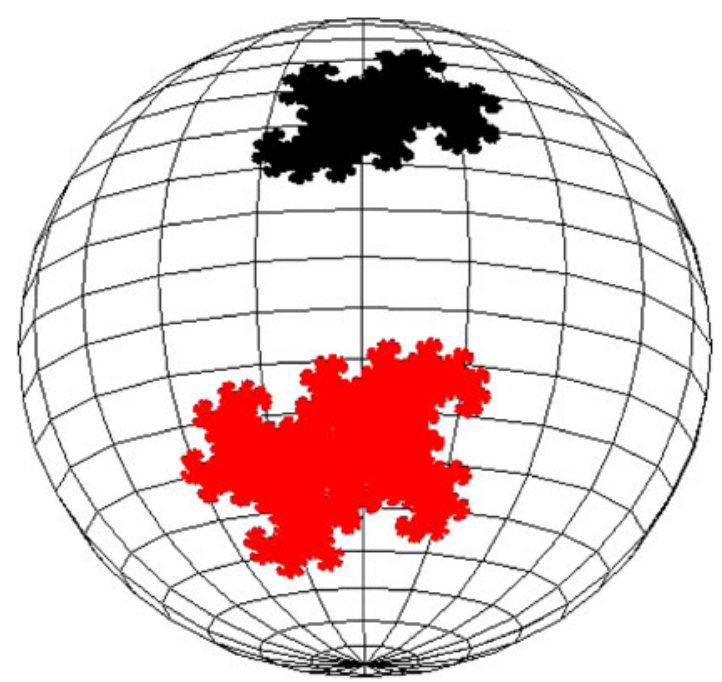

FIGURE 1. The attractor (red) and repeller (black) of a Möbius IFS consisting of two Möbius transformations. (See the online version for colours.)

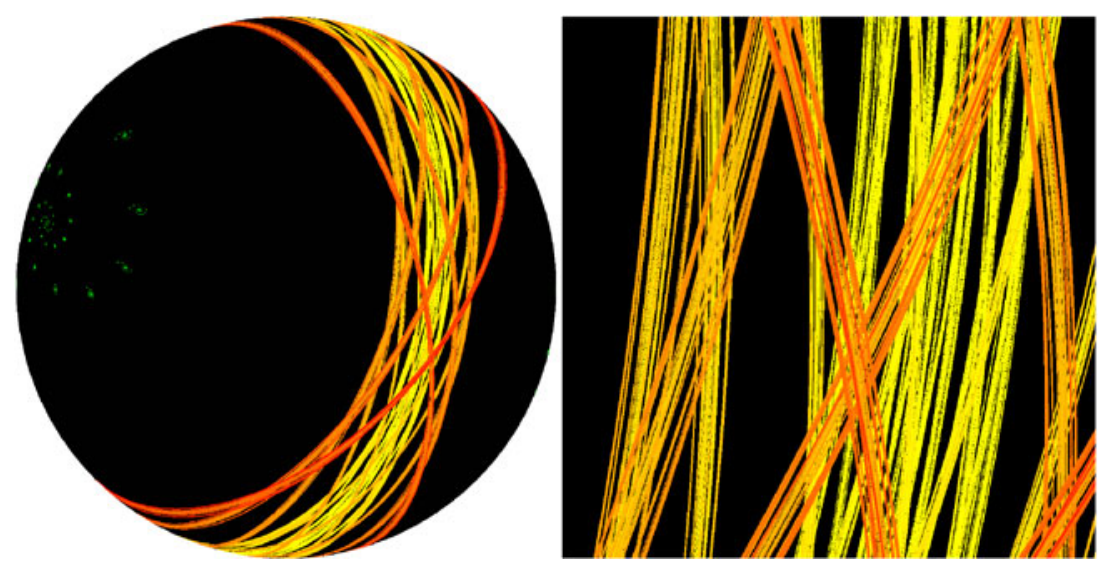

FIGURE 2. A projective attractor-repeller pair and a zoom. (See the online version for colours.)

The attractor $A$ is the union of the infinitely many red, yellow and orange lines. (See the online version for colours.) In the right panel, a zoom is shown which displays the fractal structure of the set of lines that comprise the attractor. The colours help to distinguish lines in the attractor. The corresponding dual repeller $A^{*}$ is the spiral Cantor set shown in green. The basin of $A$ is $\mathbb{R} \mathbb{P}^{2} \backslash A^{*}$. For further details on such examples, see [6].

As indicated by the proof of Theorem 4.3, an example of a noncontractive affine IFS on $\mathbb{R}^{n}$ or a Möbius IFS on $\widehat{\mathbb{C}}$ with a strict attractor cannot exist. 


\section{References}

[1] R. Atkins, M. F. Barnsley, D. C. Wilson and A. Vince, 'A characterization of point-fibred affine iterated function systems', Topology Proc. 38 (2010), 189-211.

[2] M. F. Barnsley, Fractals Everywhere (Academic Press, Boston, MA, 1988).

[3] M. F. Barnsley, 'Fractal image compression', Notices Amer. Math. Soc. 43 (1996), 657-662.

[4] M. F. Barnsley and K. Lesniak, 'On the continuity of the Hutchinson operator', arXiv:1202.2485.

[5] M. F. Barnsley and A. Vince, 'The chaos game on a general iterated function system', Ergod. Th. $\mathcal{E}$ Dynam. Sys. 31 (2011), 1073-1079.

[6] M. F. Barnsley and A. Vince, 'Real projective iterated function systems', J. Geom. Anal. 22 (2012), $1137-1172$.

[7] C. Conley, 'Isolated invariant sets and the Morse index', CBMS Regional Conference Series, 38 (American Mathematical Society, 1978).

[8] G. A. Edgar, Measure, Topology, and Fractal Geometry (Springer, New York, 1990).

[9] M. W. Hirsch and M. Hurley, 'Connected components of attractors and other stable sets', Aequationes Math. 53 (1997), 308-323.

[10] J. Hutchinson, 'Fractals and self-similarity', Indiana Univ. Math. J. 30 (1981), 713-747.

[11] A. Kameyama, Distances on Topological Self-similar Sets, Proceedings of Symposia in Pure Mathematics, 72.1 (2004).

[12] B. Kieninger, Iterated Function Systems on Compact Hausdorff Spaces (Shaker, Aachen, 2002).

[13] R. McGehee, 'Attractors for closed relations on compact Hausdorff spaces', Indiana Univ. Math. J. 41 (1992), 1165-1209.

[14] R. P. McGehee and T. Wiandt, 'Conley decomposition for closed relations', Differ. Equ. Appl. 12 (2006), 1-47.

[15] J. Milnor, 'On the concept of attractor', Commun. Math. Phys. 99 (1985), 177-195.

[16] J. Milnor, 'On the concept of attractor: correction and remarks', Commun. Math. Phys. 102 (1985), 517-519.

[17] A. Vince, 'Möbius iterated function systems', Trans. Amer. Math. Soc. 265 (2013), 491-509.

MICHAEL F. BARNSLEY, Department of Mathematics, Australian National University, Canberra, ACT, Australia e-mail: michael.barnsley@maths.anu.edu.au, mbarnsley@aol.com

ANDREW VINCE, Department of Mathematics, University of Florida, Gainesville, FL 32611-8105, USA

e-mail: avince@ufl.edu 